activity levels, however, has received less attention. Accordingly, we tested the potential usefulness of a commercially-available pedometer (NL-2000) as an outcome measure for pulmonary rehabilitation. This instrument, which is a uniaxial accelerometer, accurately detects walking activity in healthy adults.

Methods: Fifty-five patients with COPD who were referred to participating centers in the Northeast Pulmonary Rehabilitation Consortium (mean age 69 $\mathrm{yr}, \mathrm{FEV}_{1} 45 \%$ ) were studied after informed consent was obtained. All were given usual outpatient pulmonary rehabilitation, with pre- to postrehabilitation assessments of the six minute walk distance (6MWD), MRC dyspnea, and health status, using a self-administered version of the Chronic Respiratory Disease Questionnaire (CRQ). Pedometer activity was measured over a $\sim$ one-week period early in rehabilitation and again for $\sim 1$ week at the end of rehabilitation. The device was not worn during rehabilitation sessions. Patients recorded in a diary the exact times they wore the device. Pre- to postrehabilitation changes in NL-2000 counts per hour worn $\left(\mathrm{NL} \cdot \mathrm{hr}^{-1}\right)$ were evaluated, as well as the standard outcome variables (6 MWD, MRC, and CRQ).

Results: Improvements were observed in the 6MWD (45 m.), MRC dyspnea (- 0.54 units) and the CRQ total score (10 units) (all, $P<0.001)$. However, there was no change in $\mathrm{NL} \cdot \mathrm{hr}^{-1}(30$ counts, $\mathrm{I} P$. $=0.11)$. $\mathrm{NL} \cdot \mathrm{hr}^{-1}$ were lower on rehabilitation days than on nonrehabilitation days; after adding this as a covariate, changes in activity became statistically significant.

Conclusion: These results suggest that either pedometer activity assessment is relatively insensitive to detecting activity in slow-moving patients with COPD and/or pulmonary rehabilitation has less effect on increasing activity than in improving exercise performance, dyspnea, or health status.

\section{Is Prolonged and Repeated Exercise-Induced Myo- cardial Ischemic Training Deleterious?}

\author{
Noël Martin, Jean Jobin, Audrey Marcoux, Luce \\ Boyer, Gilles R Dagenais, Peter Bogaty, Institut \\ universitaire de cardiologie et de pneumologie, \\ Hôpital Laval, Québec, Qc, Canada
}

Background: In patients with ischemic heart disease (IHD), the current guidelines on exercise prescription recommend that exercise training intensity be 10 beats/min below the heart rate at which there is $>1$ mm ST-segment depression (ischemic threshold). However, it is not well established that exercise training above the ischemic threshold is harmful.

Methods: Twenty-two patients with angiographically documented IHD ( $>70 \%$ stenosis) were randomized to exercise training either at a target intensity that induced myocardial ischemia (ischemic group) or that adhered to current guidelines (control group). Training was progressively increased to 60 min under continuous ECG monitoring. Cardiac troponin T (cTnT) was measured at regular intervals. Ambulatory ECG monitoring was performed before and after 6 wkof training and left ventricular function was evaluated by echocardiography in the ischemic group after at least $6 \mathrm{wk}$ of training.

Results: The ischemic training sessions were very well tolerated. The ischemic group had myocardial ischemia during the first 20, 40, and $60 \mathrm{~min}$ exercise sessions for $12.3 \pm 6.8 \mathrm{~min}, 29.0 \pm 12.9 \mathrm{~min}$ and 49.8 $\pm 2.2 \mathrm{~min}$, respectively, with ST-segment depression ranging from 1.0 to $2.1 \mathrm{~mm}$. The estimated myocardial work (as expressed by RPP) during the training session was also higher in the ischemic than in the control group for the first $20 \mathrm{~min}$ (17 $354 \pm 6528$ vs $13355 \pm 2936$ beats/min $\bullet \mathrm{mmHg}$, respectively; $\mathrm{P}=0.08), 40 \min (16329 \pm 5407$ vs $12452 \pm 2330$ beats/min $\bullet \mathrm{mmHg}$, respectively; $\mathrm{P}=0.04)$, and $60 \mathrm{~min}$ training sessions $(18750 \pm 5698$ vs $13352 \pm 2947$ beats/min $\bullet \mathrm{mmHg}$, respectively; $\mathrm{P}=0.02$ ) No patient in either group demonstrated significant arrhythmias nor increased cTnT. The measured cTnT stayed below the detectable values of the essay $(>0.01 \mu \mathrm{g} / \mathrm{l})$ for 
all patients at all times. Left ventricular function remained unchanged in the ischemic group.

Conclusion: In patients with IHD, prolonged and repeated ischemic training sessions up to $60 \mathrm{~min}$ can be well tolerated without evidence of myocardial injury, significant arrhythmias or left ventricular dysfunction. Thus exercising at or above the ischemic threshold does not appear deleterious under this kind of supervision.

Supported by Institut de cardiologie de Québec, Inc.

\section{Taking the Stress Out of Stress Testing}

Hohenleitner Miller, Rosemary, Pennsylvania Hospital, Philadelphia, PA, USA

Background: Stress testing is a widely used screening tool for finding coronary artery disease, the leading killer of men and women in the United States. Stress tests are a safe noninvasive way to detect coronary ischemia and sift out which patients may benefit from percutaneous coronary intervention or coronary artery bypass graft. This presentation is an overview of the factors to be considered when Stress Testing.

Methods: Many factors must be considered before deciding which type of stress test best suits the patient. Time, cost and patient compliance as well as preexisting factors in their medical history such as their electrocardiogram, pacemaker or internal defibrillator, and medications. Keep in mind the following conditions make the EKG portion uninterpretable: LBBB, LVH with strain, repolarization abnormalities secondary to digitalis, baseline abnormalities, and ventricular pacing. If patients are not fit enough to perform an adequate amount of time on a treadmill due to deconditioning or musculoskeletal problems such as arthritis, a pharmacologic stress test should be performed such as a Persantine or Adenosine stress test. When patients are taking heart lowering medication like beta blockers, non-hydropyridine calcium channel blockers, digitalis, or amiodarone, they may have a blunted heart rate response making the EKG portion of the test non-diagnostic. Such patients can be instructed not to take their medication that morning or may consider having a pharmacalogic test instead.
Results: Viability studies are sometimes coupled with pharmacalogic stress tests to see if the cardiac muscle is viable and would benefit from perfusion by percutaneous intervention or bypass. These tests are performed by giving the patient sublingual nitroglycerin prior to performing the resting images in a nuclear stress test.

Conclusion: Many factors must be taken into consideration before ordering a stress test to see which one would benefit the patient the most. Keep in mind the patients physical condition, medications, and baseline EKG before choosing which one to order. 